\title{
653.
}

\section{ON A TORSE DEPENDING ON THE ELLIPTIC FUNCTIONS.}

[From the Quarterly Journal of Pure and Applied Mathematics, vol. xIv. (1877), pp. 235-241.]

ON attempting to cover with paper one half-sheet of the foregoing sextic torse, [652], I found that the paper assumed approximately the form of a circular annulus of an angle exceeding $360^{\circ}$, and this led me to consider the general theory of the construction of a torse in paper, and, in particular, to consider the torses such that when developed into a plane the edge of regression becomes a circular arc. It is scarcely necessary to remark that, to construct in paper a circular annulus of an angle exceeding $360^{\circ}$, we have only to take a complete annulus, cut it along a radius, and then insert (gumming it on to the two terminal radii) a portion of an equal circular annulus; drawing from each point of the inner circular boundary a halftangent, and considering these half-tangents as rigid lines, the paper will bend round them so as to form the half-sheet of a torse having for its edge of regression this inner boundary, which will assume the form of a closed curve with two equal and opposite maxima and two equal and opposite minima, described on a cylinder, and being approximately such as the curve given by the equations

$$
x=\cos \theta, y=\sin \theta, z=m \cos 2 \theta \text {. }
$$

Considering, in general, an arc $P Q$ (without inflexions) of any curve, and drawing at the consecutive points $P, P^{\prime}, P^{\prime \prime}$, \&c. the several half-tangents $P T, P^{\prime} T^{\prime \prime}, P^{\prime \prime} T^{\prime \prime}, \ldots$, then, considering these as rigid lines and bending the paper round them, we have the half-sheet of a torse, having for its edge of regression the curve in question now bent into a curve of double curvature. It is, moreover, clear that the edge of regression has at each point thereof the same radius of absolute curvature as the original plane curve; in fact, if in the plane curve $P P^{\prime}=d s$, and the angle $T^{\prime} P T$ between the consecutive half-tangents $P T$ and $P^{\prime} T^{\prime}$ be $=d \phi$, these quantities $d s$ and c. $\mathrm{X}$. 
$d \phi$ remain unaltered in the curve of double curvature; and the radius of absolute curvature is given by the equation $\rho d \phi=d s$. In particular when, as above, the arc is a circular one, say of radius $=\alpha$, then, however the paper is bent, the edge of regression has at each point thereof the radius of absolute curvature $=\alpha$.

Consider on any given surface, at a given point $P$ thereof, and in a given direction, an element of length $P P^{\prime}$, then (under the restrictions presently mentioned) we can determine the consecutive element $P^{\prime} P^{\prime \prime}$, such that the curve $P P^{\prime} P^{\prime \prime} \ldots$ shall have at $P$ a radius of absolute curvature $=\alpha$; in fact, $r$ being the radius of curvature of the normal section of the surface through the element $P P^{\prime}$, the radius of curvature of the section inclined at an angle $\theta$ to the normal section is $=r \cos \theta$; so that we have only to take the section at the inclination $\theta,=\cos ^{-1} \cdot \frac{\alpha}{r}$ to the normal section, and we have the consecutive element $P^{\prime} P^{\prime \prime}$ such that the radius of absolute curvature of the curve $P P^{\prime} P^{\prime \prime}$ is $=\alpha$. The necessary restriction, of course, is that $r>\alpha$; thus, if at the given point $P$ the two principal radii of curvature are of the same sign (to fix the ideas, let the two principal radii and also $\alpha$ be each of them positive), then we may on the surface determine a direction $P Q$, for which the radius of curvature of the normal section is $=\alpha$; and then the direction of the element $P P^{\prime}$ may be any direction between $P Q$ and the direction $P R$, corresponding to the greatest of the two principal radii.

Having obtained the element $P^{\prime} P^{\prime \prime}$, we may, if the radius of absolute curvature at $P^{\prime}$ be given, construct the next element $P^{\prime} P^{\prime \prime}$, and so on; that is to say, on a given surface starting from a given point $P$ and given initial direction $P P^{\prime}$, we can (under a restriction, as above, as to the curvature at the different points of the surface) construct a curve having at the successive points thereof given values of the radius of absolute curvature; viz., the value may be given either as a function of the coordinates of the point on the surface, or as a function of the length of the curve measured say from the initial point $P$; it is in this last manner that in what follows the value of the radius of absolute curvature is assumed to be given.

We may thus, taking on paper an arc $P Q$ with its half-tangents, apply it to a given surface, the point $P$ to a given point, and the infinitesimal are $P P^{\prime}$ to an element $P P^{\prime}$ in a given direction from the given point; and we thus obtain the half-sheet of a torse having for its edge of regression a determinate curve upon the surface. In particular, the arc $P Q$ may be circular of the radius $\alpha$, and the surface be a circular cylinder of radius $a$; and we thus obtain the torse having for edge of regression a curve on the cylinder radius $\alpha$, and such that the radius of absolute curvature is at each point $=a$. There are three cases according as $a>\alpha, a=\alpha$, or $a<\alpha$; it is to be remarked that if $a>\alpha$, then the curve must at each point cut the generating line of the cylinder at an angle not exceeding $\cos ^{-1} \frac{\alpha}{a}$, but that in the other two cases the angle may have any value whatever; and, further, that in every case when the angle is $=0$, viz. when the curve touches a generating line of the cylinder, then the osculating plane of the curve coincides with the tangent plane of the cylinder. 
The analytical theory is very simple. Taking $x, y, z$ as functions of the length $s$, we have

$$
\left(\frac{d x}{d s}\right)^{2}+\left(\frac{d y}{d s}\right)^{2}+\left(\frac{d z}{d s}\right)^{2}=1
$$

the condition, which expresses that the radius of absolute curvature is $=a$, then is

$$
\left(\frac{d^{2} x}{d s^{2}}\right)^{2}+\left(\frac{d^{2} y}{d s^{2}}\right)^{2}+\left(\frac{d^{2} z}{d s^{2}}\right)^{2}=\frac{1}{a^{2}}
$$

By what precedes, the point $(x, y, z)$ may be taken to be upon a given surface, say upon the cylinder $x^{2}+y^{2}=\alpha^{2}$; and we may then write $x=\alpha \cos \theta, y=\alpha \sin \theta$. Taking instead of $s$ any independent variable $u$ whatever, and using accents to denote the derived functions in regard to $u$, the equations become

$$
\begin{gathered}
x^{\prime 2}+y^{\prime 2}+z^{\prime 2}=s^{\prime 2}, \\
x^{\prime \prime 2}+y^{\prime \prime 2}+z^{\prime \prime 2}-s^{\prime \prime 2}=\frac{1}{a^{2}} s^{\prime 4}, \\
x=\alpha \cos \theta, y=\alpha \sin \theta .
\end{gathered}
$$

From the last two equations we obtain

$$
x^{\prime 2}+y^{\prime 2}=\alpha^{2} \theta^{\prime 2}, x^{\prime \prime 2}+y^{\prime \prime 2}=\alpha^{2}\left(\theta^{\prime \prime 2}+\theta^{\prime 4}\right),
$$

and the first two equations thus become

$$
\begin{gathered}
a^{2} \theta^{\prime 2}+z^{\prime 2}=s^{\prime 2} \\
a^{2}\left(\theta^{\prime \prime 2}+\theta^{\prime 4}\right)+z^{\prime \prime 2}-s^{\prime \prime 2}=\frac{1}{a^{2}} s^{\prime 4}
\end{gathered}
$$

and from the first of these we find

$$
s^{\prime \prime}=\frac{\overline{\alpha^{2} \theta^{\prime} \theta^{\prime \prime}+z^{\prime} z^{\prime \prime}}}{\left(\alpha^{2} \theta^{\prime 2}+z^{\prime 2}\right)^{\frac{1}{2}}}
$$

whence the second equation is

$$
\alpha^{2}\left(\theta^{\prime \prime 2}+\theta^{\prime 4}\right)+z^{\prime \prime 2}-\frac{\left(\alpha^{2} \theta^{\prime} \theta^{\prime \prime}+z^{\prime} z^{\prime \prime}\right)^{2}}{\left(\alpha^{2} \theta^{\prime 2}+z^{\prime 2}\right)}=\frac{\left(\alpha^{2} \theta^{\prime 2}+z^{\prime 2}\right)^{2}}{a^{2}}
$$

or reducing, this is

$$
\left(\alpha^{2} \theta^{\prime 2}+z^{\prime 2}\right)\left(\theta^{\prime \prime 2}+\theta^{\prime 4}\right)+\left(\theta^{\prime 2} z^{\prime \prime 2}-2 \theta^{\prime} \theta^{\prime \prime} z^{\prime} z^{\prime \prime}-\alpha^{2} \theta^{\prime 2} \theta^{\prime \prime 2}\right)=\frac{1}{a^{2} \alpha^{2}}\left(\alpha^{2} \theta^{\prime 2}+z^{\prime 2}\right)^{3}
$$

Taking here $\theta$ as the independent variable, we have $\theta^{\prime}=1, \theta^{\prime \prime}=0$, and the equation becomes

$$
\left(\alpha^{2}+z^{\prime 2}\right)+z^{\prime \prime 2}=\frac{1}{a^{2} \alpha^{2}}\left(\alpha^{2}+z^{\prime 2}\right)^{3} ;
$$

or, what is the same thing,

$$
z^{\prime \prime 2}=\frac{1}{a^{2} \alpha^{2}}\left(\alpha^{2}+z^{\prime 2}\right)^{3}-\left(\alpha^{2}+z^{\prime 2}\right)^{2}
$$

Write here

$$
\alpha^{2}+z^{\prime 2}=\Omega^{2},
$$


then

and the equation becomes

$$
z^{\prime \prime}=\frac{\Omega \Omega^{\prime}}{\sqrt{\left(\Omega^{2}-\alpha^{2}\right)}}
$$

or say

$$
\frac{\Omega^{\prime 2}}{\Omega^{2}-\alpha^{2}}=\frac{\Omega^{4}}{a^{2} \alpha^{2}}-1
$$

$$
\frac{a \alpha d \Omega}{\sqrt{\left(\Omega^{2}-\alpha^{2} \cdot \Omega^{4}-a^{2} \alpha^{2}\right)}}=d \theta
$$

viz. this equation determines $\Omega$ as a function of $\theta$, and we then have

$$
\left\{\begin{array}{l}
d s=\Omega d \theta \\
d z=\sqrt{ }\left(\Omega^{2}-\alpha^{2}\right) d e \\
x=\alpha \cos \theta \\
y=\alpha \sin \theta
\end{array}\right.
$$

equations which determine $x, y, z, s$ as functions of the parameter $\theta$, and give thus the edge of regression of the torse in question.

It is clear that the formulæ are very much simplified in the case $a=\alpha$, where the radius of absolute curvature $a$ is equal to the radius $\alpha$ of the cylinder; but it is worth while to develope the general case somewhat further. assume

Considering the elliptic functions $\operatorname{sn} u, \operatorname{cn} u, \operatorname{dn} u$, to the modulus $k\left(=k^{\prime}\right)=\frac{1}{\sqrt{ }(2)}$,

then

$$
\Omega=-\frac{\sqrt{ }(a \alpha)}{k} \frac{\operatorname{dn} u}{\operatorname{sn} u}
$$

$$
\begin{aligned}
d \Omega & =-\frac{\sqrt{ }(a \alpha)}{k} \frac{\mathrm{cn} u d u}{\operatorname{sn}^{2} u}, \\
\Omega^{2}-\alpha^{2} & =\frac{a \alpha}{k^{2} \operatorname{sn}^{2} u}\left(\operatorname{dn}^{2} u-\frac{\alpha}{a} k^{2} \operatorname{sn}^{2} u\right), \\
& =\frac{a \alpha}{k^{2} \operatorname{sn}^{2} u}\left\{1-\left(1+\frac{\alpha}{a}\right) k^{2} \operatorname{sn}^{2} u\right\}, \\
\Omega^{4}-a^{2} \alpha^{4} & =\frac{a^{2} \alpha^{2}}{k^{4} \operatorname{sn}^{4} u}\left(\operatorname{dn}^{4} u-k^{4} \operatorname{sn}^{4} u\right), \\
& =\frac{a^{2} \alpha^{2}}{k^{4} \operatorname{sn}^{4} u}\left(1-2 k^{2} \operatorname{sn}^{2} u\right),=\frac{a^{2} \alpha^{2}}{k^{4} \operatorname{sn}^{4} u} \operatorname{cn}^{2} u,
\end{aligned}
$$

and hence

$$
\begin{aligned}
& d \theta=\frac{k^{2} \operatorname{sn} u d u}{\sqrt{\left\{1-\left(1+\frac{\alpha}{a}\right) k^{2} \operatorname{sn}^{2} u\right\}}}, \\
& d s=\frac{k \sqrt{ }(a \alpha) \mathrm{dn} u d u}{\sqrt{\left\{1-\left(1+\frac{\alpha}{a}\right) k^{2} \operatorname{sn}^{2} u\right\}}}, \\
& d z=k \sqrt{ }(a \alpha) d u .
\end{aligned}
$$


We have thus $z=k \sqrt{ }(a \alpha) u$, no constant of integration being required, viz. $u$ is a mere constant multiple of $z$ : and the first and second equations then give $s$ and $\theta$ as functions of $u$, that is, of $z$; but it is obviously convenient to retain $u$ instead of expressing it in terms of $z$. As regards the form of these integrals observe that, writing sn $u=\lambda$, we have

and thence

$$
d u=\frac{d \lambda}{\sqrt{ }\left\{1-\lambda^{2} \cdot 1-k^{2} \lambda^{2}\right\}},
$$

$$
\begin{aligned}
& d \theta=\frac{k^{2} \lambda d \lambda}{\sqrt{\left\{1-\lambda^{2} \cdot 1-k^{2} \lambda^{2} \cdot 1-\left(1+\frac{a}{\alpha}\right) k^{2} \lambda^{2}\right\}},} \\
& d s=\frac{k \sqrt{ }(a \alpha) d \lambda}{\sqrt{\left\{1-\lambda^{2} \cdot 1-\left(1+\frac{\alpha}{a}\right) k^{2} \lambda^{2}\right\}}},
\end{aligned}
$$

each of which is in fact reducible to elliptic integrals, but I do not further pursue this general case.

In the particular case $a=\alpha$, we have

and the equations become

$$
1-\left(1+\frac{\alpha}{a}\right) k^{2} \operatorname{sn}^{2} u=\operatorname{cn}^{2} u
$$

$$
d \theta=\frac{k^{2} \operatorname{sn} u d u}{\operatorname{cn} u}, d s=\frac{k \alpha \operatorname{dn} u d u}{\operatorname{cn} u},
$$

which admit of immediate integration; viz. we have

$$
\theta=\frac{1}{2} \frac{k^{2}}{k^{\prime}} \log \frac{\mathrm{dn} u+k^{\prime}}{\operatorname{dn} u-k^{\prime}}
$$

or determining the constant so that $\theta$ may vanish for $u=0$, say

and

$$
\theta=\frac{1}{2} \frac{k^{2}}{k^{\prime}} \log \left(\frac{\operatorname{dn} u+k^{\prime}}{\operatorname{dn} u-k^{\prime}} \cdot \frac{1-k^{\prime}}{1+k^{\prime}}\right)
$$

viz. to verify these results we have

$$
s=\frac{1}{2} k \alpha \log \left(\frac{1+\operatorname{sn} u}{1-\operatorname{sn} u}\right)
$$

$$
\begin{aligned}
\frac{d \theta}{d u} & =\frac{1}{2} \frac{k^{2}}{k^{\prime}} \cdot k^{2} \operatorname{sn} u \operatorname{cn} u\left\{\frac{1}{\operatorname{dn} u+k^{\prime}}-\frac{1}{\operatorname{dn} u-k^{\prime}}\right\}, \\
& =\frac{k^{s} \operatorname{sn} u \operatorname{cn} u}{\operatorname{dn}^{2} u-k^{\prime 2}},=k^{2} \frac{\operatorname{sn} u}{\operatorname{cn} u},
\end{aligned}
$$

and

$$
\begin{aligned}
\frac{d s}{d u} & =\frac{1}{2} k \alpha \cdot \operatorname{cn} u \operatorname{dn} u\left\{\frac{1}{1+\operatorname{sn} u}+\frac{1}{1-\operatorname{sn} u}\right\}, \\
& =\frac{k \alpha \operatorname{cn} u \operatorname{dn} u}{1-\operatorname{sn}^{2} u},=\frac{k \alpha \operatorname{dn} u}{\operatorname{cn} u}
\end{aligned}
$$


Hence, recurring to the original equations, and writing for convenience $a=\alpha=1$, we see that a solution of the simultaneous equations

is

$$
\begin{aligned}
& \left(\frac{d x}{d s}\right)^{2}+\left(\frac{d y}{d s}\right)^{2}+\left(\frac{d z}{d s}\right)^{2}=1 \\
& \left(\frac{d^{2} x}{d s^{2}}\right)^{2}+\left(\frac{d^{2} y}{d s^{2}}\right)^{2}+\left(\frac{d^{2} z}{d s^{2}}\right)^{2}=1
\end{aligned}
$$

$$
\begin{gathered}
x=\cos \theta, y=\sin \theta, z=k u, \\
\theta=\frac{1}{2} \frac{k^{2}}{k^{\prime}} \log \left(\frac{\mathrm{dn} u+k^{\prime}}{\operatorname{dn} u-k^{\prime}} \cdot \frac{1-k^{\prime}}{1+k^{\prime}}\right), s=\frac{1}{2} k \alpha \log \left(\frac{1+\operatorname{sn} u}{1-\operatorname{sn} u}\right),
\end{gathered}
$$

where, as before, $k=k^{\prime}=\frac{1}{\sqrt{ }(2)}$.

Restoring the radius $\alpha$, and writing the system in the form

$$
\begin{gathered}
x=\alpha \cos \theta, y=\alpha \sin \theta, z=k \alpha u \\
\theta=\frac{1}{2} \frac{k^{2}}{k^{\prime}} \log \left(\frac{\operatorname{dn} u+k^{\prime}}{\operatorname{dn} u-k^{\prime}} \cdot \frac{1-k^{\prime}}{1+k^{\prime}}\right), s=\frac{1}{2} k \alpha \log \left(\frac{1+\operatorname{sn} u}{1-\operatorname{sn} u}\right),
\end{gathered}
$$

we see that, as $u$ passes from $u=0$ to $u=K$, and therefore $z$ from $z=0$ to $z=k \alpha K\left(K\right.$ the complete function $\left.F_{1}\left\{\frac{1}{\sqrt{(2)}}\right\}\right)$, then $\theta$ and $s$ each pass from 0 to $\infty$; and, similarly, as $u$ passes from $u=0$ to $u=-K$, that is, as $z$ passes from 0 to $-k \alpha K$, then $\theta$ passes from 0 to $\infty$, and $s$ from $s=0$ to $s=-\infty$; viz. the curve makes in each direction an infinity of revolutions about the cylinder. Developing the cylinder, $\alpha \theta$ becomes an $x$-coordinate; viz. we have thus the plane curve

$$
\begin{aligned}
& z=k \alpha u, \\
& x=\frac{1}{2} \frac{k^{2} \alpha}{k^{\prime}} \log \left(\frac{\operatorname{dn} u+k^{\prime}}{\operatorname{dn} u-k^{\prime}} \cdot \frac{1-k^{\prime}}{1+k^{\prime}}\right),
\end{aligned}
$$

which is a curve extending from the origin in the direction $x$ positive, to touch at infinity the two parallel asymptotes $z= \pm k \alpha K$; and conversely, when such a plane curve is wound about the cylinder, there will be in each direction an infinity of revolutions round the cylinder. 